\title{
Effect of inelastic scattering on the average Coulomb-blockade peak height in quantum dots
}

\author{
C W J Beenakker, ${ }^{1} \mathrm{H}$ Schomerus, ${ }^{1}$ and P G Silvestrov ${ }^{12}$ \\ ${ }^{1}$ Instituut Lorentz, Untversiteit Leiden, PO Box 9506, 2300 RA Leiden, The Netherlands \\ ${ }^{2}$ Budker Insttute of Nuclear Physics 630090 Novosibtrsk, Russia
}

(Received 25 October 2000, revised manuscript received 30 January 2001, published 13 June 2001)

\begin{abstract}
The average height of the Coulomb-blockade conductance peaks for chaotıc elastıc scattering is known to increase by a factor of $4 / 3$ upon breaking tıme-reversal symmetry We calculate the temperature dependence of this factor in the regime where the inelastic scattering rate $\Gamma_{\mathrm{m}}$ is greater than the mean tunneling rate $\Gamma_{\mathrm{el}}$, which itself is less than the mean level spacing $\Delta$ Comparison to recent experimental data by Folk et al (Folk, Patel, Marcus, Duruoz, and Harris, cond-mat/0008052) demonstrates that $\Gamma_{\mathrm{m}}$ lies below $\Gamma_{\mathrm{el}}$ and hence also below $\Delta$, consistent with the low-energy suppression of inelastic electron-electron scattering in quantum dots

DOI 10 1103/PhysRevB 64033307

PACS number(s) $7323 \mathrm{Hk}, 7350 \mathrm{Bk}, 7350 \mathrm{Gr}$
\end{abstract}

Inelastic election-electron scattering in a quantum dot broadens the single-particle excitation levels by an amount $\hbar \Gamma_{\text {in }}$ This broadening vanishes at low excitation energies $\varepsilon$ and remains less than the mean level spacing $\Delta$ as long as $\varepsilon$ 1s below the Thouless energy ${ }^{12}$ Early Coulomb-blockade experiments by Sivan et $a^{3}$ agreed with this theoretical prediction, but recent experiments by Folk et $a l^{4}$ were interpreted as inconsistent with it

Inelastic scattering can be detected by the broadening of the single-particle density of states, as was done by Sivan et al (Ref 3) Folk et al ${ }^{4}$ instead, used the temperatuie dependence of the herght of the Coulomb-blockade peaks in the conductance For fully elastic and chaotic scatteing the average height is increased by a temperature-independent factor of $4 / 3$ upon application of a magnetıc field ${ }^{56}$ Folk et al measured a suppiession of this enhancement factor when the thermal energy $k T$ became larger than $\Delta$ They concluded from this strong temperature dependence that the dephasing rate $^{7}$ in quantum dots is larger than $\Delta / \hbar$ at excitation energies well below the Thouless energy, in apparent contradıction with the theoretical expectation However, in the absence of a quantitative prediction for the temperature dependence of the Coulomb-blockade peak height, it is difficult to decide whether the observed temperature dependence is actually stronger than expected

What we will do here is use the semiclassical theory of the Coulomb blockade ${ }^{8}$ to obtain the temperature dependence in the regime $\Gamma_{\mathrm{el}} \ll \Gamma_{1 \mathrm{n}}$, with $\Gamma_{\mathrm{el}}$ the mean (elastic) tunnel rate into the quantum dot We call this the regime of strong inelastic scattermg, where "strong" means strong enough to thermalize the distribution of the electrons among the levels in the quantum dot Both $\Gamma_{\mathrm{el}}$ and $\Gamma_{\text {in }}$ should be less than $k T$, so that we are allowed to use rate equations based on sequential tunneling The condition for the Coulomb blockade is $\Gamma_{\mathrm{el}} \ll \Delta / \hbar$ and $k T \ll e^{2} / C$, with $C$ being the capacitance of the quantum dot We find that the experimental temperature dependence ${ }^{4}$ is actually much weaker than predicted by the theory for stiong inelastic scattering Therefore, $\Gamma_{\mathrm{In}} \leqslant \Gamma_{\mathrm{el}} \leqslant \Delta / \hbar$ and there is no disagieement between the experimental data of Ref 4 and the theoretical expectation of a low-energy suppiession of melastic electron-electron scattering in quantum dots ${ }^{9}$
The starting point of our analysis is a pair of expressions from Ref 8 for the $N$ th conductance peak in the two cases of putely elastic scattering $\left(G_{\mathrm{el}}\right)$ and strong inelastic scattering $\left(G_{\mathrm{nn}}\right)$

$$
\begin{aligned}
& G_{\mathrm{el}}=\frac{e^{2}}{k T} P_{\mathrm{eq}}(N)\left\langle\frac{\Gamma^{\mathrm{l}} \Gamma^{\mathrm{z}}}{\Gamma^{\mathrm{l}}+\Gamma^{\mathrm{l}}}\right\rangle_{N}, \\
& G_{\mathrm{in}}=\frac{e^{2}}{k T} P_{\mathrm{eq}}(N) \frac{\left\langle\Gamma^{\mathrm{l}}\right\rangle_{N}\left\langle\Gamma^{\mathrm{y}}\right\rangle_{N}}{\left\langle\Gamma^{\mathrm{l}}+\Gamma^{\mathrm{r}}\right\rangle_{N}}
\end{aligned}
$$

The spectral average of the elastic tunnel rate $\Gamma_{p}^{1 \mathrm{r}}$ into the left or inght reservon is defined by

$$
\left\langle\Gamma^{\mathrm{l} r}\right\rangle_{N}=\sum_{p} \Gamma_{p}^{\mathrm{l}}\left[1-F_{\mathrm{eq}}\left(E_{p} \mid N\right)\right] f\left(E_{p}-\mu\right)
$$

The equilibrium distributions $P_{\mathrm{eq}}(N)$ and $F_{\mathrm{eq}}\left(E_{p} \mid N\right)$ give, respectively, the a pilon $l$ probability to find $N$ electrons in the quantum dot and the conditional probability to find level $p$ occupied by one of the $N$ electrons (These functions are obtained from the Gibbs distribution in the canonical ensemble ) The function $f\left(E_{p}-\mu\right)$ is the Fermi-Diac distribution, with $\mu$ an externally tunable parameter that depends linearly on the gate voltage

If $\Gamma_{\text {in }} \ll \Gamma_{\text {el }}$ one may neglect inelastıc scattermg and use $\mathrm{Eq}$ (1), while if $\Gamma_{\mathrm{el}} \ll \Gamma_{\text {in }}$ one should use Eq (2) The key difference between the two equations is that for $G_{\mathrm{el}}$ the fraction $\Gamma_{p}^{\mathrm{l}} \Gamma_{p}^{\mathrm{l}} /\left(\Gamma_{p}^{\mathrm{l}}+\Gamma^{\mathrm{r}}\right)$ as a whole is averaged over the spectu um, while for $G_{\mathrm{In}}$ the numerator and denommator are averaged separately Since the spectial average extends ovel about $k T / \Delta$ levels, the difference between $G_{\mathrm{el}}$ and $G_{\mathrm{in}}$ vanishes if $k T$ becomes less than $\Delta$

In a chaotic quantum dot, the tunnel rates $\Gamma_{p}^{\mathrm{I}}$ and $\Gamma_{q}^{\mathrm{r}}$ fluctuate independently according to the Porter-Thomas distribution $P(\Gamma) \propto \Gamma^{\beta / 2-1} \exp \left(-\beta \Gamma / 2 \Gamma_{\mathrm{el}}\right) \quad$ (We assume tunneling through two equivalent single-channel point contacts, with energy-independent mean tunnel rate $\Gamma_{\mathrm{el}}$ ) The index $\beta=1$ (2) in the presence (absence) of a time-reversalsymmetry bieaking magnetic field The mean height $\bar{G}_{\mathrm{el}}^{\overline{\mathrm{max}}}$ of the Coulomb-blockade peak for elastic scattering increases upon breaking tıme-reversal symmetıy, by a temperature- 


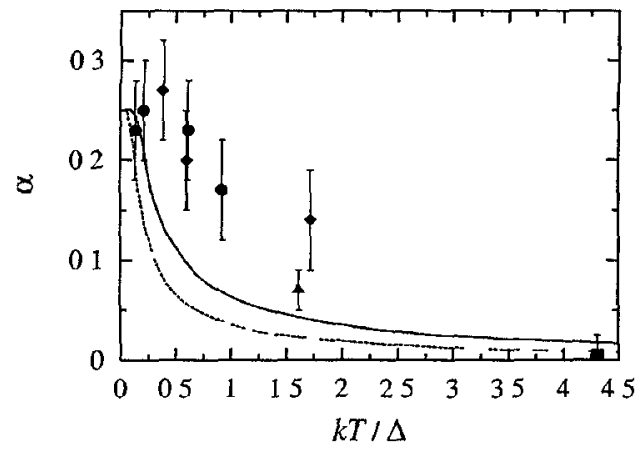

FIG 1 Temperature dependence of the parameter $\alpha$ defined in Eq (4) The curves are calculated from Eq (2), etther for spin degenerate levels (solid) or for nondegenerate levels (dashed) The markers with error bars are experimental data for GaAs quantum dots from Folk et al (Ref 4) The area of the dot is 025 0 7, 3, and $8 \mu \mathrm{m}^{2}$ for, respectively, circle, diamond, triangle, and square markers

independent factor of $4 / 3^{56}$ Inelastic scattering introduces a temperature dependence, which we can study using Eq (2)

Qualitatively, the effect of inelastic scattering on the 4/3enhancement factor can be understood as follows The spectial average \langle\rangle$_{N}$, defined precisely in $\mathrm{Eq}$ (3), can be approximated by an average over $k T / \Delta$ levels around the Fermi energy in the quantum dot contaning $N$ elections If $k T \gg \Delta$ the spectral average becomes equivalent to an ensemble average The ensemble averages of $\Gamma_{p}^{l}$ and $\Gamma_{p}^{\prime}$ ate both equal to the $\beta$-independent value $\Gamma_{e l}$, so the peak height (2) for strong inelastic scattering simplifies to $G_{1 \mathrm{n}}$ $\approx \frac{1}{2} \Gamma_{\text {el }}\left(e^{2} / k T\right) P_{\text {eq }}(N)$ - independent of whether timereveisal symmetry is broken or not This explans why the enhancement factor drops from $4 / 3$ to 1 as $k T$ becomes larger than $\Delta$ in the case of stiong inelastic scattering

For a quantitative comparison, we have plotted in Fig 1 the temperature dependence of the parameter

$$
\alpha=1-\bar{G}_{\mathrm{nn}}^{\overline{\max }}(\beta=1) / \bar{G}_{\mathrm{in}}^{\overline{\max }}(\beta=2),
$$

which diops from $1 / 4$ to 0 as $k T$ becomes largei than $\Delta$ The solid curve is for equally-spaced spin degenerate levels $\left(E_{2 p}=E_{2 p-1}=p \Delta, \Gamma_{2 p}=\Gamma_{2 p-1}\right)$ Because the spin degeneracy might be lifted spontaneously, ${ }^{10}$ we also show for comparison the case of equally spaced nondegenerate levels
( $E_{p}=p \Delta / 2$, all $\Gamma_{p}$ 's independent) In either case $\Delta$ is defined as the mean level spacing of a single spin degree of freedom We see that the temperature dependence is stronger for nondegenerate levels An even stronger temperature dependence (not shown) is found if, instead of equally spaced levels, we would use a Wigner-Dyson distribution The data points are the experimental results of Folk et al,${ }^{4}$ for GaAs quantum dots of four different areas The values of $\Delta$ used are those given in Ref 4 , estimated from the area $A$ and the twodimensional density of states $\left(\Delta=2 \pi \hbar^{2} / m A\right.$, with $m$ the effective mass of the electrons) There is therefore no adjustable parameter in the comparison between theory and experiment

It is clear from $F_{1} g$ i that the experimental temperature dependence is much weaker than the theoretical piediction, regardless of whether we include spin degeneracy or not We have found that the theory would fit the data within the error bars if we would rescale $k T / \Delta$ by a factor of 3 (with spin degeneracy) or a facto1 of 5 (without spin degeneracy) Such a laige factor is beyond the experimental uncertanty in level spacing or temperature We conclude that the inelastic scattering rate is well below $\Gamma_{\text {el }}$ and $\Delta / \hbar$ for a range of energies within $k T$ One possible explanation of the deviation of our theoretical curves from the experimental data would be that only the high-lying levels have equilibiated, while the lowlying levels have not Such an explanation would be consistent with the scenario put forwaid in Ref 2 , according to which the discieteness of the spectrum pievents the lowlying levels to equilibrate on an arbitiarily long time scale

We conclude with two suggestions for future reseatch on this topic From the theoretical side, it would be useful to generalize Ref 8 to an arbitiaty 1 atio of $\Gamma_{\mathrm{el}}$ and $\Gamma_{\mathrm{in}}$ [going beyond the two limits of laige and small $\Gamma_{\mathrm{el}} / \Gamma_{\text {in }}$ given in Eqs (1) and (2)] From the expermental side, it would be of interest to compare data for the temperature dependence of $\alpha$ for different values of $\Gamma_{\mathrm{el}}$, that is to say, for different heights of the tunnel barriels separating the quantum dot fiom the election reseivois We would expect the data points in Fig 1 to approach the theoretical curves as the tunnel baniers are made higher and higher, giving mote piecise information on the rate of melastic scattering

This work was supported by the Dutch Science Foundation NWO/FOM We have benefited from corlespondence with P W Brouwei and C M Marcus
${ }^{1} \mathrm{U}$ Sivan, Y Imry, and A G Aronov, Europhys Lett 28, 115 (1994)

${ }^{2}$ B L Altshuler, Y Gefen, A Kamenev, and L S Levitov, Phys Rev Lett 78, 2803 (1997)

${ }^{3} U$ Sivan, F P Milliken, K Milkove, S Rishton, Y Lee, J M Hong, V Boegl, D Kern, and $M$ de Franza, Europhys Lett 25, 605 (1994)

${ }^{4} \mathrm{~J}$ A Folk, S R Patel, C M Marcus C I Duruoz, and J S Harris, Jr , cond-mat/0008052 (unpublished)

${ }^{5} \mathrm{R}$ A Jalabert, A D Stone, and Y Alhassid, Phys Rev Lett 68 ,
3468 (1992)

${ }^{6}$ Y Alhassid, Phys Rev B 58, R 13383 (1998)

${ }^{7}$ Folk et al (Ref 4) interpret their data in terms of the dephasing rate $\Gamma_{\phi}$, but we would argue that therr experiment is more sensitive to the inelastic scattering rate $\Gamma_{m}$ than to $\Gamma_{\phi}$ It is melastic scattering that destroys the $\frac{4}{3}$-enhancement of the conductance peaks - not dephasing Indeed, the theories of Refs 4-6 use a model of "sequential tunneling," in which there is no phase coherence at all between the electron entering and leaving the quantum dot It is irrelevant for the $\frac{4}{3}$ enhancement whether 
such phase coherence exists or not One can conclude from the experimental data that $\Gamma_{\mathrm{m}} \leqslant \Gamma_{\mathrm{el}}, \Gamma_{\phi} \ll \Delta / \hbar$, but the relatıve magnitude of $\Gamma_{\mathrm{el}}$ and $\Gamma_{\phi}$ remains undetermined

${ }^{8}$ C W J Beenakker, Phys Rev B 44, 1646 (1991)
${ }^{9} \mathrm{~A}$ similar analysis of the experiment has been proposed independently by $\mathbf{P}$ W Brouwer (private communication)

${ }^{10}$ Ya M Blanter, A D Mirlın, and B A Muzykantskıı, Phys Rev Lett 78, 2449 (1997) 